\title{
A Physics-Based Pattern Dictionary for EBSD Image Segmentation
}

\author{
Se Un Park ${ }^{1}$, Dennis Wei ${ }^{1}$, Marc De Graef ${ }^{2}$, Megna Shah $^{3}$, Jeff Simmons ${ }^{3}$, Alfred O. Hero ${ }^{1}$ \\ ${ }^{1}$ Dept. of Electrical Engineering \& Computer Science, University of Michigan, Ann Arbor, MI, USA. \\ 2 Dept. of Materials Science and Engineering, Carnegie Mellon University, Pittsburgh, PA, USA. \\ ${ }^{3}$ Materials and Manufacturing Directorate, Air Force Research Laboratory, Dayton, OH, USA.
}

We describe a new segmentation and anomaly detection method for electron backscatter diffraction (EBSD) images. In contrast to conventional methods that require Euler angles to be extracted from diffraction patterns, the proposed method operates on the patterns directly. We use a forward model implemented as a dictionary of diffraction patterns generated by a detailed physics-based simulation of EBSD. This allows us to exploit information that is normally discarded in the indexing process, in particular the characteristics of the background intensity profile. With this additional information, grain boundaries and anomalous points can be detected as explicit classes at the same time as grains are segmented. Anomaly detection is an important capability since anomalies may correspond to defects or contaminants in the material that affect its properties. In addition, processing the diffraction patterns directly avoids problems with indexing when the observed pattern is a poor match to the dictionary, as occurs for example at grain boundaries.

The dictionary of 281700 diffraction patterns is generated using a computational physics-based forward model. For each orientation, the process consists of three steps. The first step models the interaction of the electron beam with the sample using the Schrodinger equation with a Bloch wave ansatz [1]. The backscattered electron yield is calculated for a set of directions covering the hemisphere of all possible exit directions. The second step is to interpolate the intensities over the hemisphere onto the pixel locations on the collecting detector. We make use of an equal-area projection of the hemisphere onto a square or hexagonal grid [2] to permit standard bilinear interpolation of the intensities. The third step is to model additional instrument effects such as detector quantum efficiency, Poisson noise, coupling optics, and the point spread function and binning mode of the CCD. The final result is a dictionary of 281700 diffraction patterns, which we represent as a $480 \times 281700$ matrix with each column corresponding to a vectorized $80 \times 60$ pixel diffraction pattern.

To demonstrate the proposed approach the physics-based pattern dictionary was generated for the Nibase alloy IN100. Fig. 1 compares a simulated pattern from the dictionary to two measured patterns collected at AFRL from an IN100 sample. To segment and classify pixels in the EBSD image we used a decision tree (DT) [3], shown in Fig. 2, that classifies pixels as grains interiors, edges, shifted background anomalies, and noisy background anomalies (shown from right to left at the leaves of the DT in the Figure). The DT structure is a hybrid classification tree in the sense that it uses different similarity measures at each node to perform the classification. The two measures are: (1) normalized inner products between a measured pattern and the patterns in the dictionary and (2) the number of common dictionary indices among the top 40 dictionary nearest neighbors of each pixel in a $3 \times 3$ neighborhood centered at the pixel of interest. Similarity measure (1) is applied to the raw patterns while similarity (2) is applied to background compensated patterns. The only tuning parameters in the DT classifier are the decision thresholds on the similarity measures used at each node of the tree. Based on a physics based model, the tree structure of our DT classifier is fixed and need not be learned from the data.

Fig. 3(a) shows the segmented image produced by our DT classifier. In terms of segmenting grains, our result is consistent with the image in Fig. 3(b) from a conventional Euler-angle based segmentation al- 
gorithm, as implemented in DREAM.3D [4]. However, the proposed method also identifies anomalies (colored red and blue in Fig. 3(a)) that are either not detected (black clusters) or misclassified as one or more grains in Fig. 3(b). This is due to the fact that the conventional segmentation algorithm is not aware of anomalies and has difficulty extracting meaningful Euler angles from anomalous diffraction patterns, as indicated by the black clusters.

\section{References}

[1] A Winkelmann et al, Ultramicroscopy 107 (2007), p. 414-421.

[2] D Roşca, Astronomy and Astrophysics 520 (2010), p. 1-4.

[3] J Friedman et al, “The Elements of Statistical Learning” (Springer Series in Statistics, 2009).

[4] DREAM.3D, http://dream3d.bluequartz.net, accessed February 5, 2013

Figure 1: Background-compensated experimental patterns for (a) interior and (b) edge pixels. Best-match dictionary element (c) from our forward model for both (a) and (b).
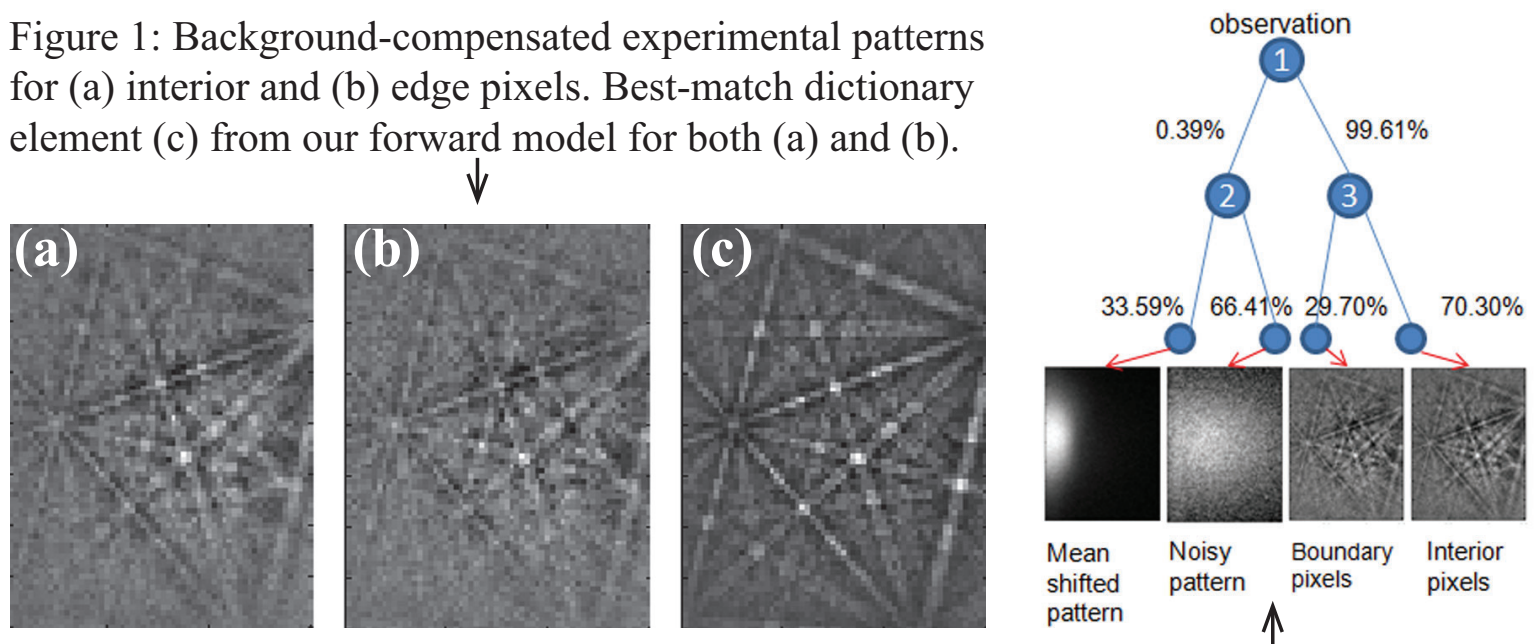

Figure 2: Decision tree. The background similarity criterion (1) is used at nodes 1 and 2 while the neighborhood similarity criterion (2) is used at node 3 . The division of the population at each parent node is shown above the branches. Representative diffraction patterns are also shown. The percentages indicate the division of the parent population at each node, e.g. $99.61 \%$ of the pixels in the sample were classified as having non-anomalous background patterns (two right-most patterns in the figure).
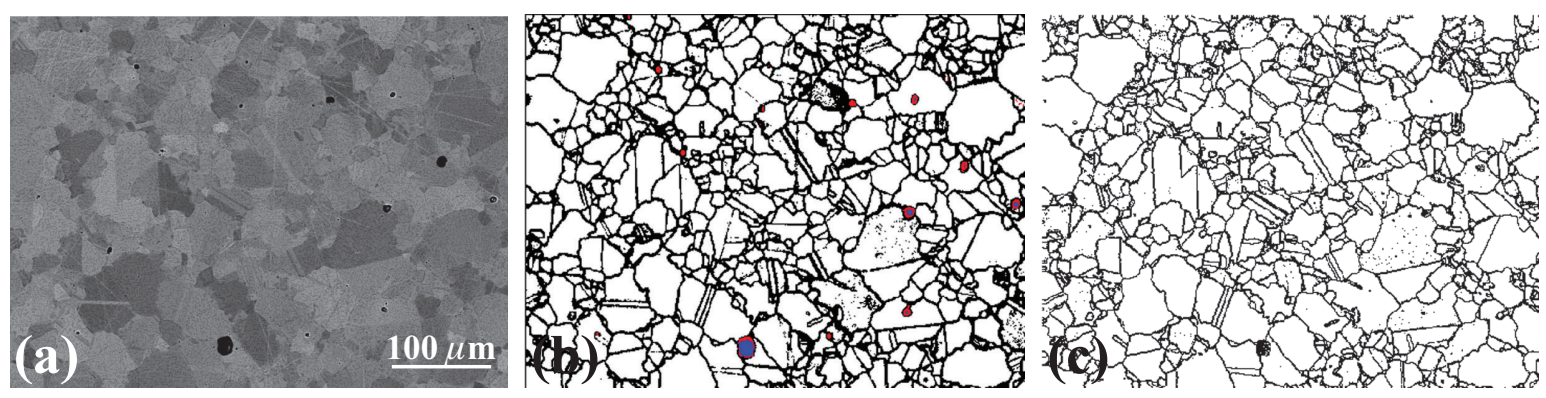

Figure 3: (a) back-scattered electron image; (b) segmentation result from our decision tree in Fig. 2. Grain interiors in white, boundaries in black, noisy pixels in red, and backgroundshifted pixels in blue. (c) segmentation result from standard extracted Euler-angle method (implemented in DREAM.3D). Black clusters in the right image represent pixels that cannot be classified by standard methods but that are classified as specific types of background anomalies by the proposed DT method. 